\title{
Substrate reduction therapy as a new treatment option for patients with Gaucher disease type 1: A review of literatures
}

\author{
Young Bae Sohn ${ }^{1}$ and Han-Wook Yoo ${ }^{2 * *}$ \\ ${ }^{1}$ Department of Medical Genetics, Ajou University Hospital, Ajou University School of Medicine, Suwon, Korea \\ ${ }^{2}$ Department of Pediatrics, Asan Medical Center Children's Hospital, University of Ulsan College of Medicine, Seoul, Korea
}

\begin{abstract}
Gaucher disease type 1 (GD1) is an inherited lysosomal storage disorder caused by deficiency of acid $\beta$-glucosidase. The diminished enzyme activity leads to the accumulation of substrates and results in multi-systemic manifestations including hepatosplenomegaly, anemia, thrombocytopenia, and bone diseases. Enzyme replacement therapy (ERT) by infusion of recombinant protein has been the standard treatment for over 20 years. Despite the successful long-term treatment with ERT, several unmet needs remain in the treatment of GD1 such as severe pulmonary and skeletal manifestations. Substrate reduction therapy (SRT) reduces the accumulation of substrates by inhibiting their biosynthesis. Eliglustat, a new oral SRT, was approved in United States and Europe as a first-line therapy for treating adult patients with GD1 who have compatible CYP2D6 metabolism phenotypes. Although eliglustat is not yet available in Korea, introduction and summary of this new treatment modality are provided in this paper by review of literatures. Despite the fact that there are only limited studies to draw resolute conclusions, the current data demonstrated that eliglustat is not inferior to ERT in terms of its clinical efficacy. The approval of eligustat enables eligible adult GD1 patients to have the option of oral therapy although it still needs further studies on long-term outcomes. The individual patient should be assessed carefully for the choice of treatment modality when eliglustat becomes available in Korea. Furthermore, the clinical guidelines for Korean patients with GD1 regarding the use of eliglustat needs to be developed in near future.
\end{abstract}

Key words: Gaucher disease, Substrate reduction therapy, Enzyme replacement therapy, Eliglustat.

\section{Introduction}

Gaucher disease type 1 (GD1, OMIM230800) is an autosomal recessively inherited lysosomal storage disorder caused by deficiency of acid $\beta$-glucosidase, which is responsible for the degradation of glucosylceramide and glucosylsphingosine [1]. Thus, the deficiency of the enzyme results in the accumulation of glucosylceramide and glucosylsphingosine in lysosomes of tissue macrophages and consequently leads to multisystemic manifestations. Clinical symptoms of GD1 include hepatosplenomegaly, anemia, thrombocytopenia, pulmonary diseases, immune dysfunction and bone disease. The diagnosis of GD1 is confirmed by demonstrating decreased activity of acid $\beta$-glucosidase in leukocytes and/or by molecular analysis of the

\footnotetext{
Received: 1 December 2016, Revised: 15 December 2016, Accepted: 20 December 2016, Published: 31 December 2016

*Corresponding author: Han-Wook Yoo, M.D., Ph.D.

Department of Pediatrics, Asan Medical Center Children's Hospital, University of Ulsan College of Medicine, 88 Olympic-ro 43-gil, Songpa-gu, Seoul 05505, Korea.

Tel: +82-2-3010-3374, Fax: +82-2-473-3725, E-mail: hwyoo@amc.seoul.kr

Conflict of interest: The authors declare that they do not have any conflicts of interest.

(c) This is an open-access article distributed under the terms of the Creative Commons Attribution Non-Commercial License (http://creativecommons.org/licenses/by-nc/4.0/) which permits unrestricted non-commercial use, distribution, and reproduction in any medium, provided the original work is properly cited.

(c) Copyright 2016 by the Korean Society of Medical Genetics

www.e-kjgm.org
} 


\section{GBA gene [2].}

For treatment of GD1, enzyme replacement therapy (ERT) with intravenous infusion of recombinant acid $\beta$-glucosidase has been the standard treatment for more than two decades. ERT has proven to improve systemic manifestations of GD1 by degradation of accumulated glucosylceramide [2-4]. Despite the successful long-term treatment with ERT, there are still several unmet needs in the treatment of GD1 that continue to exist. Some patients experience skeletal symptoms or severe pulmonary symptoms which are refractory to ERT [5].

Substrate reduction therapy (SRT) reduces the production of the substrates by inhibiting glucosylceramide synthase, the rate-limiting step of glucosylceramides synthesis, thereby decreasing the over-production and accumulation of substrates [6]. The drugs for SRT are administered orally in contrast to intravenous ERT. The first developed oral SRT drug for treatment of GD1 was miglustat (Zavesca ${ }^{\circledR}$; Actelion Pharmacueticals, Allschwil, Switzerland), which was approved for mild to moderately affected GD1 patients who cannot receive ERT $[7,8]$. Unlike miglustat which is a second-line therapy to ERT, eliglustat (Cerdelga ${ }^{\circledR}$; Sanofi Genzyme, Cambridge, MA, USA) was approved by the Food and Drug Administration (FDA; 2014) and the European Medicines Agency (EMA; 2015) as a firstline treatment for adult patients with GD1 who are CYP2D6 extensive, intermediate, or poor metabolizers ( $>90 \%$ of patients) [9-11]. The recommendations of eliglustat treatment are also recently published by advisory council of experts in US and Europe $[10,11]$.

Although ERT for patients with GD1 has been used successfully for more than 20 years in Korea, the physicians have no clinical experience with eligustat as it is not yet available in Korea. The aim of this paper is to introduce the new treatment modality using eliglustat in managing patients with GD1 by review of literatures. This review could help to provide guidelines for the selection of treatment options of Korean GD1 patients in near future.

\section{Enzyme Replacement Therapy}

ERT has been treatment of choice for over 20 years since it has been available in early 1990s. Three recombinant acid $\beta$-glucosidase products are approved for the treatment of GD1; imiglucerase (Cerezyme ${ }^{\circledR}$; Sanofi Genzyme), velaglucerase alpha (VPRIV ${ }^{\circledR}$; Shire Human Genetic Therapies, Lexington, MA, USA), and taliglucerase alpha (ELELYSO ${ }^{\circledR}$, Pfizer Labs, New York, NY, USA). In Korea, the non-comparable biologic imiglucerase (Abcertin ${ }^{\circledR}$; ISU Abxis, Seoul, Korea) is also available since its approval by Korean Ministry of Food and Drug Safety in 2012 [12]. ERT is administered by intravenous infusion of $60 \mathrm{U} / \mathrm{kg}$ of drug every other week. ERT leads to breakdown of stored glucosylceramide and can achieve amelioration of hepatosplenomegaly, anemia, thrombocytopenia, and bone manifestations [2-4]. Longitudinal data from International Collaborative Gaucher Group (ICGG) Gaucher Registry demonstrated that most of the hematologic and visceral benefits occur during the first year of ERT, with maintained or sustained improvement in all parameters for at least 8 years of treatment $[2,13]$. Although approximately $7 \%$ of patients have experienced recurrent mild adverse events, the majority of adverse events were mild infusion-related reactions including itching and urticaria during infusions, which could be easily controlled by slowing the infusion rate and using antihistamine or corticosteroid [2]. Nonetheless, there are several limitations of ERT that entail a great burden for patients due to nature of life-long intravenous injections. First of all, the inconvenience of every other week infusion can interrupt social activities of the patients and reduce quality of life. The expensive medical costs also should be considered. Furthermore, ERT cannot completely prevent severe pulmonary or bone complications despite the long-term treatment [14].

\section{Substrate Reduction Therapy}

SRT aims to reduce accumulating glucosylceramide and related materials by inhibiting their synthesis. Comparing with recombinant proteins used in ERT, inhibitors of glycosylceramide synthesis used in SRT are small molecules that can be taken orally and potentially diffuse into various tissues, including the central nervous system and bones [15].

Miglustat, the first developed SRT drug, has been available in both Europe and US since its approval in 2002 and 2003, respectively [11]. Significant improvement in hepatosplenomegaly and biochemical markers has been demonstrated with miglustat treatment $[7,8,16]$. However, miglustat, iminosugar of a synthetic analog of D-glucose, is a non-selective inhibitor of glycosidases as well as an inhibitor of glucosylceramide synthase and causes considerable side effects including gastrointestinal complaints (abdominal cramping and diarrhea) by inhibiting intestinal glycosidases. These complications have led many patients to discontinue the treatment $[7,8]$, designating miglustat as a second-line therapeutic option for limited cases who cannot receive ERT [11]. 
Although miglustat can cross the blood brain barrier in murine models, improvements of neurological manifestations in type IIII $\mathrm{GD}$ are controversial $[15,16]$. It remains as the first-line drug for Niemann-Pick type $\mathrm{C}$ disease.

Eliglustat, a new selective glycosylceramide synthase inhibitor, was recently approved as a first-line therapy for adult patients with GD1 by FDA and EMA $[10,11]$. Eliglustat has stronger inhibitory potency than miglustat [15]. In contrast to miglustat, eliglustat does not cause gastrointestinal side effect because it is not a potent inhibitor of intestinal glycosidases that prevents off-target actions.

Phase II and III clinical trials demonstrated that eligustat significantly reduces spleen and liver volumes, increases levels of hemoglobin and platelet counts compared with placebo in treatment-naïve patients with GD1 $[17,18]$ and maintains long-term stability $[17,19]$. In patients whose disease status had been stabilized with ERT, a switch to eliglustat treatment showed non-inferiority compared with ERT [20]. Although eliglustat has been well-tolerated in clinical trials, the long-term observation for monitoring of adverse event is necessary for this newly approved drug. Commonly reported adverse events include headache, migraine, arthralgia, nausea, abdominal pain, diarrhea, and dizziness which were mild to moderate in severity [10]. As the effect of eliglustat on possible long-term complications of GD such as multiple myeloma, hematologic malignancies, Parkinsonism and peripheral neuropathy is currently undetermined [10], the long-term follow up is needed.

\section{Eliglustat Dosing and Drug Interactions}

Dosing of eliglustat is determined based on the patient's genetic CYP2D6 metabolizer status because eliglustat is extensively metabolized by CYP2D6, and less extensively, CYP3A [11]. There are four main phenotypes of CYP2D6 metabolizers poor, intermediate, extensive, and ultra-rapid. A recommended dose has not been determined for ultra-rapid metabolizers and eliglustat therapy is not recommended in these paients currently [11]. Therefore, CYP2D6 genotyping is necessary to determine the patient's eligibility and dosing. More than $90 \%$ of Caucasian population is known to be extensive or intermediate metabolizer. However, the distribution of CYP2D6 polymorphism is not well known in Korean population. Lee et al. [21,22] reported that approximately $98 \%$ of Koreans are extensive or intermediate metabolizer among 400 Koreans assessed in the study. Futhermore, if the patient uses concomitant drugs that can affect CYP2D6 or CYP3A activity, the dose of eliglustat is recommended to be adjusted. The recommendations for eliglustat standard dosing and recommendations with concomitant drugs are summarized in Table 1.

The use of eliglustat in patients with renal insufficiency or cardiac conditions has not been studied yet and eliglustat is not recommended in patients with such underlying diseases [11]. Also, the use in a pregnant or lactating woman with GD is not recommended either [11].

\section{Future Research Aspect of Substrate Reduction Therapy}

Additional clinical trials are warranted to characterize the efficacy of eliglustat in extended population cohort such as patients aged 65 years and older and children. As children were not included in the clinical trials, the additional studies are

Table 1. Recommended eliglustat dosing based on CYP2D6 metabolizer status and concomitant drug use (based on US recommendation)

\begin{tabular}{|c|c|c|c|}
\hline & \multicolumn{3}{|c|}{ CYP2D6 metabolizer status } \\
\hline & Extensive & Intermediate & Poor \\
\hline Standard dose & $84 \mathrm{mg}$ twice daily & $84 \mathrm{mg}$ twice daily & $84 \mathrm{mg}$ once daily \\
\hline \multicolumn{4}{|l|}{ Concomitant use of eliglustat with } \\
\hline $\begin{array}{l}\text { Strong or moderate CYP2D6 inhibitors+strong } \\
\text { or moderate CYP3A inhibitors }\end{array}$ & Contraindicated & Contraindicated & $\begin{array}{l}\text { Contraindicated (See recommendation } \\
\text { for strong or moderate CYP3A } \\
\text { inhibitors in poor metabolizers }{ }^{2} \text { ) }\end{array}$ \\
\hline Strong CYP2D6 inhibitors & $84 \mathrm{mg}$ once daily & $84 \mathrm{mg}$ once daily & Contraindicated (84 mg once dailyª) \\
\hline Moderate CYP2D6 inhibitors & 84 mg once daily (Cautionª) & 84 mg once daily (Caution ${ }^{\mathrm{a}}$ ) & Contraindicated (84 mg once dailyª) \\
\hline Strong CYP3A inhibitors & 84 mg once daily (Caution ${ }^{a}$ ) & Contraindicated (Caution') & Contraindicated \\
\hline Moderate CYP3A inhibitors & 84 mg once daily (Cautionª) & Not recommended (Cautionª) & Not recommended \\
\hline Weak CYP3A inhibitors & $84 \mathrm{mg}$ twice daily & $84 \mathrm{mg}$ twice daily & Not recommended (Cautionª) \\
\hline Strong CYP3A inducers & Not recommended & Not recommended & Not recommended \\
\hline
\end{tabular}

${ }^{2}$ This table is based on US recommendation [11]. European recommendations [10] are noted in parenthesis in cases of discordance. 
required to evaluate the efficacy, safety, and dosing strategies in pediatric population. Moreover, additional research is needed in CYP2D6 ultra-rapid metabolizers, who are currently not eligible for eliglustat therapy.

Potential advantages of SRT modalities over unmet needs of ERT are considered to be better outcomes of bone complications due to easier drug delivery to bones [15]. This issue needs to be clearly demonstrated by long-term clinical trial.

Although eliglustat crosses the blood-brain barrier, it is immediately transported back out of the brain by the multidrug transporter Pgp-1 [23]. This suggests that eliglustat would be ineffective for treating neuronopathic GD. Therefore, research for developing new small molecule which can be distributed into brain would be warranted.

\section{Comparison of Substrate Reduction Therapy with Enzyme Replacement Therapy}

Although the randomized controlled studies for direct comparison of eliglustat treatment with ERT has not been properly performed yet, two recent reports demonstrated the comparative outcomes of SRT and ERT. Ibrahim et al. [24] compared the clinical response to eliglustat in 46 treatmentnaïve patients ( 26 patients' data from phase II and 20 patients' data from phase III ENGAGE clinical trials) with 75 imiglucerasetreated patients (data from ICGG Gaucher registry) by posthoc comparison. The authors demonstrated that the degree of improvement in organ volumes and hematologic parameters from baseline was similar between eliglustat-treated patients and imiglucerase-treated patients during the initial 9 to 12 months of treatment [24]. Smid et al. [15] also demonstrated that biochemical markers including chitotriosidase, CCL18 and glucosylphingosine decreased comparably in small number of patients receiving eliglustat treatment (among six patients, four were treatment naïve and two were switched from ERT) and ERT $(n=4)$. At this moment, the limited studies are insufficient to conclude resolutely whether ERT and SRT using eliglustat are equivalent in clinical efficacy and safety. Nonetheless, at least several data show that SRT using eliglustat is not inferior to ERT in terms of reduction of liver and spleen volume, and improvements in hemotologic and biochemical parameters. The extended comparative studies on both SRT and ERT in larger

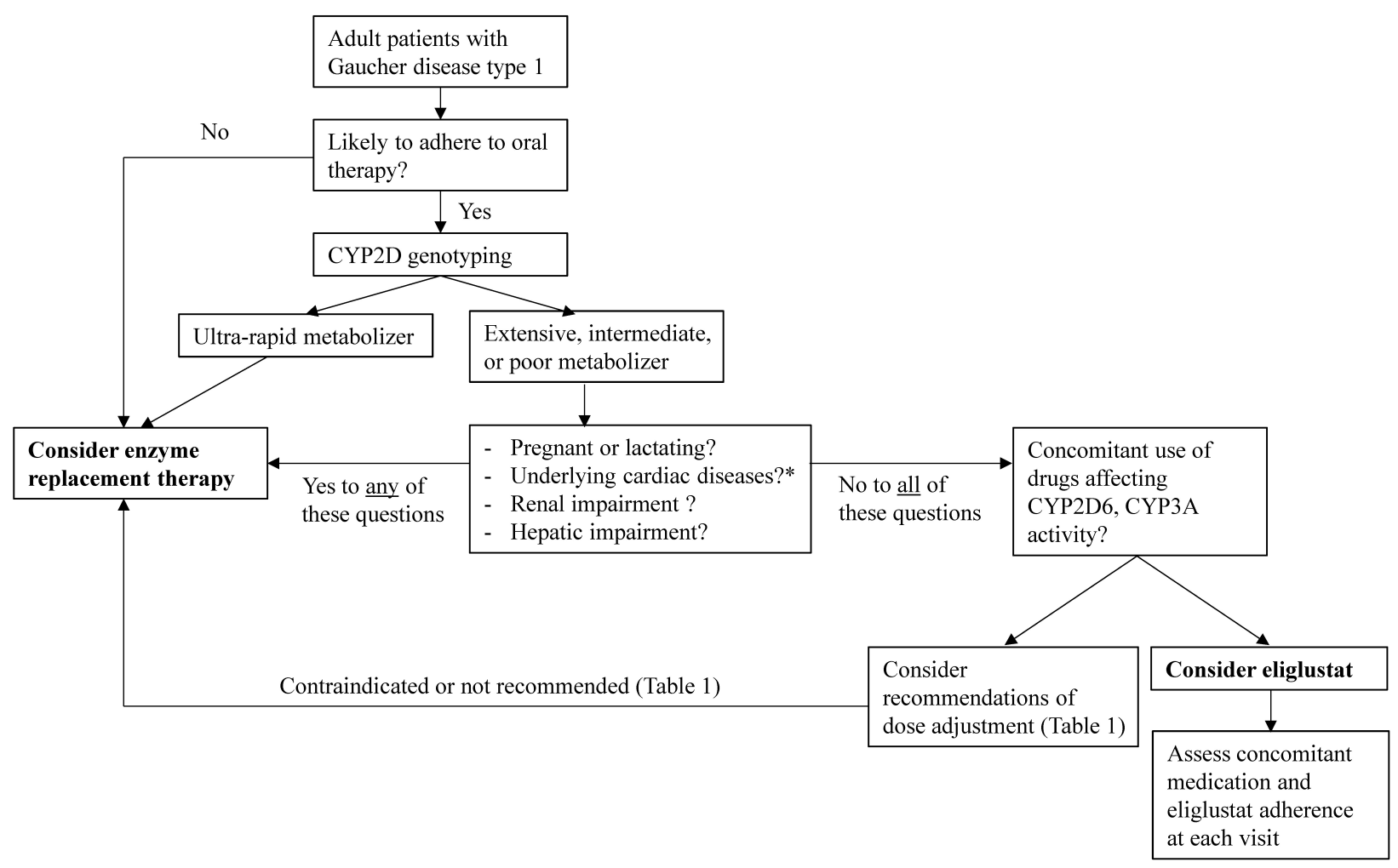

Fig. 1. Suggested algorithm to determine eligibility of eliglustat therapy in adults with Gaucher disease type 1. Modified from the article of Balwani et al. (Mol Genet Metab 2016;117:95-103) [10]. *Long QT syndrome, use of Class IA, IC or III antiarrhythmic agents, congestive heart failure, recent acute myocardial infarction, bradycardia, heart block, ventricular arrhythmia. 
cohort are needed to determine optimal clinical guidelines for treatment of patients with GD1.

\section{Deciding Choice of Therapy}

SRT with eliglustat approved as a first-line therapy for eligible patients with GD1 provides a convenient daily oral therapy as an alternative to ERT in Europe and US [10,11]. Although eliglustat is not yet available in Korea, the physicians and patients would consider the eligibility of this new treatment modality as it can be available in near future. The decision to choice of therapy should be based on the patient characteristics, underlying conditions, individual patient's needs and/or preferences for therapy, and access to each type of therapy [10]. For example, eligustat is currently approved only for adult GD1 patients older than 18 years, whereas ERT is approved for children as well as adults. Therefore, symptomatic children should be started on ERT because early treatment of symptomatic patients can improve outcomes [2]. Balwani et al. [10] suggested algorithm for determining eligibility of eligulstat therapy in adults with GD1 (Fig. 1).

\section{Conclusion}

ERT has been the mainstay of treatment in GD1 patients for more than 20 years. However, there are unmet needs in ERT. The approval of eligustat as a first-line therapy enables eligible adult GD1 patients to have the option of oral therapy although it still needs further studies on long-term outcome. Therefore, physicians should carefully assess individual patient to determine the choice of treatment modality and appropriateness of the therapy when eliglustat becomes available. Furthermore, the clinical guidelines for Korean patients with GD1 regarding the SRT using eliglustat, including starting therapy and monitoring patients, needs to be developed in near future.

\section{Acknowledgements}

This study was supported by the new faculty research fund of Ajou University School of Medicine.

\section{References}

1. Grabowski GA, Andria G, Baldellou A, Campbell PE, Charrow J, Cohen IJ, et al. Pediatric non-neuronopathic Gaucher disease: presentation, diagnosis and assessment. Consensus statements. Eur J Pediatr 2004;163:58-66

2. Kaplan $P$, Baris $H$, De Meirleir $L$, Di Rocco $M$, El-Beshlawy $A$, Huemer $M$, et al. Revised recommendations for the management of Gaucher disease in children. Eur J Pediatr 2013;172:447-58.

3. Chen M, Wang J. Gaucher disease: review of the literature. Arch Pathol Lab Med 2008;132:851-3.

4. Grabowski GA. Phenotype, diagnosis, and treatment of Gaucher's disease. Lancet 2008;372:1263-71.

5. Cox TM. Eliglustat tartrate, an orally active glucocerebroside synthase inhibitor for the potential treatment of Gaucher disease and other Iysosomal storage diseases. Curr Opin Investig Drugs 2010;11:116981.

6. Shayman JA. Eliglustat tartrate: glucosylceramide synthase inhibitor treatment of type 1 gaucher disease. Drugs Future 2010;35:613-20.

7. Cox $T$, Lachmann $R$, Hollak $C$, Aerts J, van Weely $S$, Hrebicek $M$, et al. Novel oral treatment of Gaucher's disease with N-butyldeoxynojirimycin (OGT 918) to decrease substrate biosynthesis. Lancet 2000:355:1481-5.

8. Cox TM, Amato D, Hollak CE, Luzy C, Silkey M, Giorgino $R$, et al. Evaluation of miglustat as maintenance therapy after enzyme therapy in adults with stable type 1 Gaucher disease: a prospective open-label non-inferiority study. Orphanet J Rare Dis 2012;7:102.

9. Hicks JK, Swen JJ, Thorn CF, Sangkuhl K, Kharasch ED, Ellingrod $\mathrm{VL}$, et al. Clinical Pharmacogenetics Implementation Consortium guideline for CYP2D6 and CYP2C19 genotypes and dosing of tricyclic antidepressants. Clin Pharmacol Ther 2013;93:402-8.

10. Balwani M, Burrow TA, Charrow J, Goker-Alpan O, Kaplan P, Kishnani PS, et al. Recommendations for the use of eliglustat in the treatment of adults with Gaucher disease type 1 in the United States. Mol Genet Metab 2016;117:95-103.

11. Belmatoug $N_{1}$ Di Rocco $M$, Fraga $C_{1}$ Giraldo $P_{\text {, Hughes }}$, Lukina $E_{1}$ et al. Management and monitoring recommendations for the use of eliglustat in adults with type 1 Gaucher disease in Europe. Eur Intern Med 2016, in press.

12. Choi JH, Lee $B H$, Ko JM, Sohn $Y B$, Lee JS, Kim GH, et al. A phase 2 multi-center, open-label, switch-over trial to evaluate the safety and efficacy of Abcertin ${ }^{\circledR}$ in patients with type 1 Gaucher disease. $J$ Korean Med Sci 2015;30:378-84.

13. Andersson H, Kaplan P, Kacena K, Yee J. Eight-year clinical outcomes of long-term enzyme replacement therapy for 884 children with Gaucher disease type 1. Pediatrics 2008;122:1182-90.

14. van Dussen $L$, Biegstraaten $M$, Dijkgraaf MG, Hollak CE. Modelling Gaucher disease progression: long-term enzyme replacement therapy reduces the incidence of splenectomy and bone complications. Orphanet J Rare Dis 2014;9:112. 
15. Smid BE, Ferraz MJ, Verhoek $M$, Mirzaian M, Wisse $P$, Overkleeft HS, et al. Biochemical response to substrate reduction therapy versus enzyme replacement therapy in Gaucher disease type 1 patients. Orphanet J Rare Dis 2016;11:28.

16. Brand M, Muller A, Alsop J, van Schaik IN, Bembi B, Hughes D. Results from a 9-year Intensive Safety Surveillance Scheme (IS(3) ) in miglustat (Zavesca( $\left.{ }^{\circledR}\right)$ )-treated patients. Pharmacoepidemiol Drug Saf 2015;24:329-33.

17. Lukina $E$, Watman $N$, Arreguin EA, Dragosky $M$, lastrebner $M$,

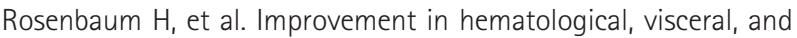
skeletal manifestations of Gaucher disease type 1 with oral eliglustat tartrate (Genz-112638) treatment: 2-year results of a phase 2 study. Blood 2010;116:4095-8.

18. Mistry PK, Lukina $E$, Ben Turkia $H$, Amato $D$, Baris $H$, Dasouki $M$, et al. Effect of oral eliglustat on splenomegaly in patients with Gaucher disease type 1: the ENGAGE randomized clinical trial. JAMA 2015;313:695-706

19. Lukina E, Watman N, Dragosky M, Pastores GM, Arreguin EA,

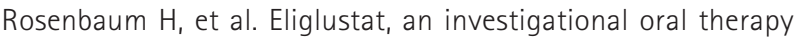
for Gaucher disease type 1: Phase 2 trial results after 4 years of treatment. Blood Cells Mol Dis 2014;53:274-6.

20. Cox TM, Drelichman G, Cravo R, Balwani M, Burrow TA, Martins $A M$, et al. Eliglustat compared with imiglucerase in patients with Gaucher's disease type 1 stabilised on enzyme replacement therapy: a phase 3, randomised, open-label, non-inferiority trial. Lancet 2015;385:2355-62.

21. Lee SJ, Lee SS, Jung HJ, Kim HS, Park SJ, Yeo CW, et al. Discovery of novel functional variants and extensive evaluation of CYP2D6 genetic polymorphisms in Koreans. Drug Metab Dispos 2009;37:1464-70.

22. Lee SY, Sohn KM, Ryu JY, Yoon YR, Shin JG, Kim JW. Sequence-based CYP2D6 genotyping in the Korean population. Ther Drug Monit 2006;28:382-7.

23. Shayman JA. The design and clinical development of inhibitors of glycosphingolipid synthesis: will invention be the mother of necessity? Trans Am Clin Climatol Assoc 2013;124:46-60.

24. Ibrahim J, Underhill LH, Taylor JS, Angell J, Peterschmitt MJ. Clinical response to eliglustat in treatment-naïve patients with Gaucher disease type 1: Post-hoc comparison to imiglucerase-treated patients enrolled in the International Collaborative Gaucher Group Gaucher Registry. Mol Genet Metab Rep 2016;8:17-9. 